(Mittheilung aus dem Institut für allgem. Pathologie u. Therapie der Universität Budapest. Director: Prof. A. Högyes ${ }^{1}$ ).)

\title{
Ueber den Zusammenhang zwischen der Muskulatur und dem Labyrinth.
}

Von

Dr. Georg v. Marikovszky, Assistent.

Mit 2 Textfiguren.

Es ist eine allbekannte Thatsache, dass zwischen dem Gehörorgan resp. dem Labyrinth und der gesammten Muskulatur ein gewisser Zusammenhang vorhanden ist.

Flourens${ }^{2}$ ) bemerkte im Jahre 1824, dass der Kopf solcher Tauben, deren halbkreisförmige Canäle er durchschnitten bat, auf höhere Töne mit krampfartigen Zuckungen reagirte. Er war jedoch noch der Meinung, dass diese Erscheinung auf das krankhaft gesteigerte Hörvermögen zurückgeführt werden kann. („L'audition douleureuse".)

Esser beobachtete im Jahre 1827, dass Hunde mit langen, hängenden Ohren diese auf hohe Geigentöne spitzten.

Breuer ${ }^{3}$ ) (1875) fasst die an operirten Thieren beobachteten pendelnden Kopf bewegungen und den Manegegang als directe Folgen einer Verletzung der halbkreisförmigen Canäle auf.

Cyon ${ }^{4}$ ) sah (1876) Pupillenverengerung auf der durch Ton gereizten und Pupillenerweiterung auf der entgegengesetzten Seite.

1) Theile der Resultate dieser Untersuchungen wurden der ungarischen Akademie der Wissenschaften am 15. Mai 1899, am 16. Juni 1902 und am 19. Januar 1903 vorgelegt.

2) Flourens, Mémoires présentés à l'Académie royale des sciences. 1824 .

3) Breuer, Beiträge zur Lehre vom statischen Sinn. 1875.

4) Cyon, Physiol. Beziehungen zwischen dem Gehörnerv und dem oculomotorischen Apparat. Compt. rend. 1876. 
Im Jahre 1877 sah $\mathrm{Cy}_{0} \mathrm{n}^{1}$ ) nach Durchschneidung der halbkreisförmigen Canäle bilaterale Augenbewegungen auftreten.

Preyer ${ }^{2}$ ) gelang es (1881), bei Meerschweinchen mit Tönen des Cri-Cri synchrone Ohrbewegungen hervorzurufen.

Mit den nystagmischen Augenbewegungen befasste sich Högyes ${ }^{3}$ ) bereits am Ende der siebziger Jahre. Seine Aufmerksamkeit wurde durch einen Zufall auf dieselben gelenkt. Er wollte nämlich untersuchen, ob das Drehen einen Einfluss auf die Mastdarmtemperatur der Thiere ausüben würde, und nahm bei dieser Gelegenheit die auftretenden Augenbewegungen wahr. (Solche Augenbewegungen konnte er übrigens auch durch das Ausreissen des Nervus facialis hervorrufen.) Als er die compensatorischen Augenbewegungen im Jahre 1879 näher untersuchte, bemerkte er, dass solche nicht nur an den Augen, sondern auch an den Augenlidern, Ohren, am Gesicht, am Nacken, in gewissem Grade sogar an der gesammten Körpermuskulatur hervorgerufen werden können. Die compensatorischen Augenbewegungen waren bei Meerschweinchen und bei Vögeln schwächer

1) Cy on, Recherches expér. sur les fonctions des canaux semicirculaires etc. Acad. d. scienc. Séance du 31 Déc. 1877.

2) Preyer, Die Seele des Kindes. 1881.

3) Högyes hat sich mit der Physiologie des Ohrlabyrinths und hauptsächlich mit dem Zusammenhang des Labyrinths und der Augenbewegungen besonders in den achtziger Jahren des vorigen Jahrhunderts sehr eingehend beschäftigt. Seine erste Mittheilung über diese Frage erschien im Jahre 1879, die letzte im Jahre 1886. In diesem Zeitraum veröffentlichte er 22 Arbeiten über die Physiologie des Ohrlabyrinths. Der grösste Theil erschien leider nur in ungarischer Sprache, einige wurden jedoch auch in deutschen Zeitschriften veröffentlicht, so "Ueber die Veränderungen des Auges nach Facialisexstirpation." Arch. f. exper. Pathol. u. Pharm. Bd. 11. - „Ueber die Wirkung einiger chemischer Stoffe auf die associirten Augenbewegungen." Arch. f. exper. Pathol. u. Pharm. Bd. 16. - „Ueber die wahren Ursachen der Schwindelerscheinungen bei der Drucksteigerung in der Paukenhöhle." Pflüger's Arch. Bd. 26. Die Resultate seines in drei Theilen erschienenen Hauptwerkes "Ueber den Nerrenmechanimus der associirten Augenbewegungen" wurden durch Prof. Klug im Biol. Centralblatt 1881 Nr. 7 und im Jahresbericht f. d. Fortschritte d. Anat. u. Physiol. Bd. 9 S. 123. 1881 auf's Ausführlichste besprochen. Da aber in diesem Referat seine Methodik nicht ausführlich beschrieben wurde, führte dies zu vielen Missverständnissen von Seite der Forscher, die seine Experimente wiederholen wollten. In neuester Zeit veröffentlichte er wieder einige kleine Arbeiten; ein grösseres Werk, das auch die Resultate neuerer Untersuchungen und die ausführliche Methodik seines Verfahrens beschreiben und auch deutsch erscheinen wird, wird gegenwärtig vorbereitet. 
als bei den Kaninchen und wurden durch Kopfbewegungen ersetzt. Im Jahre 1880 und 1881 hat Högyes den engen Zusammenhang zwischen den Augenmuskeln und dem Labyrinth auf's Ausführlichste durchforscht und beschrieben. Seine Versuche bewiesen, dass die Musc. rect. lat., obl. sup. und rect. sup. des Auges mit dem Labyrinth derselben Seite, die Musc. rect. med., obl. inf. und rect. inf. dagegen mit dem der entgegengesetzten Seite in Zusammenhang stehen. Bei Unversehrtheit beider Labyrinthe, resp. bei vollkommenen Functioniren dieser, beobachtete er an den Augen der Kaninchen während des Drehens an einer Drehscheibe einen, der Ebene des Drehens entsprechenden, bilateralen, associirten Augennystagmus. Nach Entfernung oder Zerstörung beider Labyrinthe bleiben die compensatorischen Augenbewegungen gänzlich aus, falls aber nur ein Labyrinth entfernt oder zerstört wurde, so trat Strabismus beider Augen auf. Zerstörte er z. B. nur das linke Labyrinth, so kommen am linken Auge die Musc. rect. med., obl. inf. und rect. inf., am rechten Auge die mm. Rect. lat., Obl. sup. und Rect. sup. in Contractur; die übrigen drei-drei Muskeln der beiden Augen erschlaffen dagegen vollkommen.

Im Jahre 1881 behauptete Högyes, dass im Mittelhirn und im verlängerten Mark ein Reflexcentrum vorhanden sei, dessen centripetale Bahnen die in den Labyrinthen endenden Nervi acustici, und dessen centrifugale Babnen die in den zwölf Augenmuskeln endenden motorischen Nerven bilden. Im Jahre 1882 fand er, "dass zwischen den Vertibularenden des Acusticus und gewisser Muskelcomplexe eine nähere Verbindung besteht," und weiter: „In den häutigen Bogengängen der beiden Ohrlabyrinthe mit dem Utriculus besteht eine zweifache centripetale Endvorrichtung des bilateral eingerichteten, die gesammte Körpermuskulatur associirenden oder coordinirenden Nervenapparates, welche je nach den Aenderungen ihrer Lage im Labyrinthe die unwillkürlichen bilateralen Körperbewegungen regeln."

Högyes untersuchte im Jahre 1884 in Gemeinschaft mit La uffenauer hystero-epileptische Kranke, wobei er an denselben sowohl im wachen als auch im hypnotischen Zustande folgende Beobachtungen machen konnte.

Verschliesst man das eine Ohr der Kranken mit Baumwolle und lässt dann vor dem anderen eine Stimmgabel ertönen, so geräth der Fuss der dem freien Ohre entsprechenden und der Arm der 
entgegengesetzten Seite in Contractur. Sind beide Ohren der liegenden Patientin frei, und ertönt die Stimmgabel vor ihr, so gerathen alle Extremitäten in Contractur. Wenn die stehende Kranke dem Klopfen eines Inductionsapparates eine Zeit lang zuhörte, so erstarrten die Muskeln der Arme und die des Nackens. Wenn nun ein hoher Harmonikaton ertönte, so liess die Starrbeit der Nackenmuskulatur nach, die der Arme blieb aber unverändert. Verschliesst man das eine $\mathrm{Ohr}$ der sitzenden Kranken und lässt nun vor dem freien Ohr rhythmische Musik ertönen, so fiel die untere Extremität der dem freien Ohre entsprechenden und der Arm der entgegengesetzten Seite in rhythmische Zuckungen. Wird nun die Musik plötzlich unterbrochen, bleiben die steinhart gewordenen Extremitäten in der Lage, welche sie im Momente des Unterbrechens eingenommen hatten ${ }^{1}$ ). "Es ist," sagt Högyes, „demnach bewiesen, dass der Endapparat des Acusticus eigentlich der centripetale Endapparat eines Nervenmechanismus ist, welcher nicht bloss die Augenbewegungen, sondern auch die Bewegungen des ganzen Körpers associirt."

Högyes setzte seine Versuche an Hysteroepileptikern im folgenden Jahre fort, wobei er zu dem Schlusse kam, dass von dem Gehörnerven Reflexe auf sämmtliche spinale motorische Nerven übertragen werden können.

In einer Abhandlung aus dem Jahre 1886 erwähnt Högyes seine bereits vor Jahren gemachte folgende Beobachtung. Wird vor einer Schaar Meerschweinchen in die Hände geklatscht, auf eine Flasche oder auf einen Tisch geschlagen oder auf irgend eine andere Weise ein schärferes Geräusch hervorgerufen, so werden synchron mit dem Geräusch sämmtliche Meerschweinchen Reflexzuckungen der Ohren aufweisen.

Lichtwitz ${ }^{2}$ ) (1887) sah nach grösserem Geräusch bei Hysterisehen Convulsionen auftreten.

$\mathrm{Zoth}^{3}$ ) (1901) ist der Meinung, dass die japanischen Tanzmäuse ihr Gleichgewicht ganz gut behalten können, und wenn unter gewissen Verbältnissen dies nicht der Fall zu sein scheint, so darf

1) Es ist interessant, dass solche Reflexcontracturen auch durch Tast-, Geschmack-, Geruch- und optische Reize hervorgerufen werden konnten.

2) Lichtwitz, Anaesth. hyst. Paris 1887.

3) Zoth, Ein Beitrag zu den Beobachtungen und Versuchen an japanischen Tanzmäusen. Pflüg ej's Arch. 1901. 
dies nicht (wie $\mathrm{Cy}_{0} \mathrm{n}^{1}$ ) meinte) dem Gesichtsschwindel zugeschrieben werden, sondern der unvollkommenen Innervation der Muskulatur resp. der Unvollkommenheit der Coordination.

Zwei Preise, welche die medicinische Facultät der Budapester Universität auf die Empfehlung Prof. Högyes' behufs Fortsetzung der Untersuchungen über die Physiologie des Ohrlabyrinthes ausgeschrieben hat, regten mich noch im Jahre $1897 \mathrm{zu}$ Untersuchungen auf diesem Gebiete an. Ich machte meine Versuche an Raben und Elstern, vorwiegend aber an Tauben und Kaninchen. Bei den Versuchen an Tauben verfuhr ich im grossen Ganzen nach der Methode Ewald's. Die Untersuchungen an Kaninchen machte ich nach der Methode und unter der Aufsicht Högyes', wobei ich mich überzeugt habe, dass man hierbei weniger "künstlerische Fertigkeit" (wie das einige Forscher am Anfange der achtziger Jahre bebauptet und die Resultate Högyes' in Folge dessen mit einer gewissen Skepsis empfangen haben) als vielmehr Uebung, Geduld und einer entsprechenden Leitung bedarf.

Zur Stütze meiner Folgerungen werde ich auch die diesbezüglichen Resultate der Versuche Ewald 's und Dreyfuss' erwähnen.

In erster Reihe wiederbolte ich Högyes' Versuche über den Nervenmechanismus der associirten Augenbewegungen. Die Resultate stimmten mit denen von Högyes vollkommen überein, wesshalb ich auf die nähere Beschreibung dieser verzichten kann.

Zum Theil die Versuche Ewald's wiederholend, konnte ich an Tauben, deren linkes Labyrinth ich entfernt habe, Folgendes beobachten:

1. Der Kopf der Taube ist vom dritten Tage an nach der Operation bis ungefähr zum 14. derart verdreht, dass das Schädeldach den Boden berührt, das rechte Auge nach vorne, das linke nach hinten sieht.

2. Drehe ich das Thier auf einer Drehscheibe in horizontaler Ebene nach rechts, so pendelt der Kopf nur nach dem Einstellen des Drehens. Drehe ich sie hingegen nach links, so pendelt der Kopf um die verticale Achse nur während des Drehens.

3. Beim Gehen schwankt die Taube häufig nach links, da der linke Fuss (was Cyon schon im Jahre 1878 beschrieben hat) oft einknickt.

1) Cyon, Ohrlabyrintb, Raumsinn und Orientirung. Pflüger's Arch. 1900. 
4. Die Taube dreht sich oft nach links, da sie mit dem rechten Fuss zu grosse Schritte macht.

5. Stellt sie sich auf einen Fuss, so ist das gewöhnlich der rechte.

6. Will sie sich von einer über ihren Kopf gezogenen Kappe befreien, so stellt sie sich auf das rechte Bein und kratzt die Kappe wiederholt mit dem linken.

7. Will sie sich $a b$ und $z u$ bloss auf den linken Fuss stellen, so knickt dieser ein.

8. Mit dem rechten Fuss hebt sie das daran gebundene Gewicht höher wie mit dem linken.

9. Setze ich sie auf meinen Finger, so umklammert sie denselben mit dem rechten Fuss zwar ungeschickt, aber bedeutend stärker als mit dem linken.

10. Hänge ich sie mit den Füssen an eine Schnur, so kann sie nicht fliegen; die Schläge des rechten Flügels sind zu stark; sie dreht sich in Folge dessen nach links. Wird sie bei dieser Gelegenheit erschöpft, so

11. zieht sie den rechten Flügel an den Körper, den linken lässt sie hängen. Der Schwanz wird etwas nach rechts zu gehalten ${ }^{\mathbf{1}}$ ).

12. Inducirten Strömen gegenüber zeigen sich der rechte Flügel und der rechte Fuss weniger empfindlich als die linken Extremitäten.

Von den an linksseitig operirten Tauben gemachten, hierher gehörenden Versuchen Ewald's ${ }^{2}$ ) will ich hier folgende erwähnen.

13. Hält man mit der einen Hand die Füsse, mit der anderen den Schnabel der Taube und spannt das Thier auf diese Weise in horizontale Lage, so erreicht bei den Flügelschlägen die rechte Flügelspitze die den Schnabel haltende Hand.

14. Ewald durchschnitt den unteren Schnabel links operirter Tauben in der Längsrichtung und liess die zwei Hälften nicht zusammenwachsen. Band er jetzt gleiche Gewichte an beide Schnabelhälften, so erwies sich die linke als schwächer.

Im Jahre 1896 hat $\mathrm{E}_{\mathrm{wa}} \mathrm{d}^{3}$ ) links operirte Tauben am Schwanze aufgehängt und bemerkte beim Eintritte der Todtenstarre,

1) Eine gesunde Taube wird unter solchen Umständen beide Flügel an den Körper ziehen und den Schwanz gerade halten.

2) Ewald, Physiol. Untersuchungen über das Endorgan d. Nervus octavus. Wiesbaden 1892.

3) Ewald, Die Beziehungen d. Tonuslabyr. zur Todtenstarre. Pflüger's Arch. 1896. 
15. dass sich der Kopf des Thieres um ungefähr $80^{\circ} \Varangle$ rechts drehte, wobei das linke Auge höher zu stehen kam, ferner dass sich das linke Bein und der linke Flügel ein wenig eingezogen hatten.

An Kaninchen, deren linkes Labyrinth ich nach der Methode Högyes' entfernt oder zerstört hatte, bemerkte ich Folgendes ${ }^{1}$ ):

16. Sobald das linke Labyrinth seine Function einbüsst, so dreht sich der Kopf um die verticale und um die Längsachse nach links.

17. Beide vorderen Extremitäten werden nach rechts gehalten.

18. Binde ich das Thier von dem Hög y e s'schen ${ }^{2}$ ) Kaninchenbrett los, so wälzt es sich nach links.

19. Auf der Wirbelsäule tritt eine seitliche Krümmung auf. Die langen Muskeln der rechten Seite des Rumpfes sind in starker Contractur, die der linken vollkommen erschlafft, in Folge dessen wird die Wirbelsäule nach rechts concav.

20. Drehe ich das Thier in der horizontalen Ebene nach rechts, so pendelt der Kopf nur nach dem Einstellen des Drehens. Drehe ich es dagegen nach links, pendelt der Kopf nur während des Drehens.

21. Die Haltung des Kopfes nach links und die Haltung der vorderen Extremitäten nach rechts kann man durch Drehen in verschiedenen Ebenen nur in beschränktem Maasse corrigiren.

23. Die rechte Seite des ganzen Thieres ist inducirten Strömen gegenüber weniger empfindlich als die linke.

Dreifuss ${ }^{3}$ ) sah bei linkerseits operirten Meerschweinchen ebenfalls Linksdrehung des Kopfes und Linkswälzung des Körpers. Seine Beobachtungen differiren insoweit von den meinigen, als er bei seinen halbseitig operirten Meerschweinchen die Concavität der Wirbelsäule der beschädigten Seite zugekehrt fand. Ich konnte bei meinen zahlreichen, an Kaninchen ausgeführten Versuchen immer nur das Gegentheil wahrnebmen.

Bei Tauben, deren sämmtliche Bogengänge, sei es durch Ex-

1) Natürlich habe ich die halbseitigen Operationen an Tauben und Kaninchen nicht nur links, sondern auch rechts ausgeführt; die Erscheinungen traten demgemäss natürlicher Weise in verkehrter Weise auf. Bloss der leichteren Uebersicht wegen habe ich nur die Resultate linksseitiger Operation beschrieben.

2) Högyes, Bemerkungen über die Methode der Mastdarmtemperaturbestimmung bei Thieren u. s. w. Arch. f. exper. Pathol. u. Pharm. Bd. 13.

3) Dreyfuss, Experimenteller Beitrag zur Lehre von den nicht-akustischen Functionen d. Ohrlabyrinthes. Pflüger's Arch 1900. 
stirpation, sei es durch die $\mathrm{Ewald}$ 'sche Plompirungsmethode, ausgeschaltet waren, konnte ich folgende Beobachtungen machen.

1. Ungeordnete Bewegungen des Kopfes um die eine oder andere Achse.

2. Falls in der späteren Zeit sich bei der Drehung des Thieres compensatorisehe Kopfbewegungen zeigen, sind dieselben von sehr geringer Ausdehnung und vollständig umgeordnet.

3. Diese Kopf bewegungen bleiben sowohl während als auch nach dem Drehen dieser Tauben vollkommen aus, wenn sie mit einer Kappe versehen werden.

4. Sie können die Erbsen aus einer gefüllten Schale nur nach vielen zwecklosen Kopfbewegungen aufpicken.

5. Wird die Taube geschüttelt, so baumelt ihr Kopf schlaff hin und her.

6. Die Körperhaltung beim Gehen ist wackelig, da bald das eine, bald das andere Bein einknickt.

7. Von Zeit zu Zeit geschieht eine Wendung nach rechts oder nach links, je nachdem sie mit dem rechten oder dem linken Fusse zu stark ausschreitet. Diese Erscheinung ist noch nach Jahren am

8. schlängelnden Gang zu erkennen.

9. Setzt man die Taube auf einen Stab, so ergreift sie diesen sehr ungeschickt, besonders wenn sie die Kappe auf dem Kopfe hat. (Sie tritt mit dem einen Fuss auf den anderen u. s. w.)

10. Auf den Rücken gelegt kann sie nur schwer aufstehen.

11. Ungeordnete $Z$ wangsbewegungen um eine der Körperachsen nach rechts uder nach links, nach vorne oder nach hinten sind noch nach geraumer Zeit nach der Operation wahrnehmbar.

12. Falls sie während dieser Zwangsbewegungen ermüdet, ruht sie, besonders wenn sie die Kappe auf hat, in sehr unbequemer Körperhaltung aus.

13. Wird sie an den Füssen aufgehängt, lässt sie beide Flügel schlaff herabhängen.

14. Fliegen kann sie anfangs überhaupt nicht, später auch nur sehr unvollkommen.

15. Nach beiderseitiger Exstirpation (jedoch nicht nach Plombirung) findet man die Reflexerregbarkeit beider Flügel und Füsse herabgesetzt.

Von meinen an beiderseitig operirten Kaninchen gemachten Beobachtungen will ich hier folgende anführen. 
16. Wird das Thier nach der Operation vom Kopfhalter befreit, so hält es den Kopf wohl in der Mittellinie, aber ohne Kraft, wobei das Kinn auf das Brett des Kaninchenhalters gestutzt ist.

17. Wird das Thier mit freiem Kopf auf einer Drehscheibe befestigt, so folgt der Kopf bei den Drehungen nur den Gesetzen der Schwere.

18. Es werden zwar beide vorderen Extremitäten symmetrisch und zur Mittellinie des Körpers parallel gehalten, aber so kraftlos, dass bei passiver Bewegung derselben absolut kein Widerstand wahrnehmbar ist. Ueberhaupt ist

19. die gesammte Körpermuskulatur schlaff.

20. Das Thier kann nicht aufrecht stehen, da alle vier Füsse zusammenknicken.

21. Das beiderseitig operirte Kaninchen kann nicht schlingen. Diese Thiere müssen künstlich - mit Schlundsonde oder durch den Mastdarm - ernährt werden.

22. Die Reflexerregbarkeit inducirten Stromschlägen gegenüber ist an beiden Körperhälften herabgesetzt.

Folgende einschlägige Beobachtungen, die D reyfuss an beiderseits operirten Meerschweinchen machte, gehören auch hierher.

23. Im Augenblicke der Zerstörung des zweiten Labyrinthes war die Erschlaffung der gesammten Muskulatur fühlbar.

24. Mit der Erschlaffung der Athmungsmuskeln tritt Dyspnoë auf, und in Folge der Schwächung der Phonationsmuskeln wird auch 25. die Stimme schwächer.

Aus allem dem ergibt sich nicht bloss das, dass zwischen dem Labyrinthe und der Muskulatur überhaupt ein Zusammenhang besteht, es lässt sich vielmehr auch die Art des Zusammenhanges eruiren.

In Analogie mit der Högyes'schen Entdeckung, dass je drei Augenmuskeln mit dem Labyrinthe derselben und je drei mit dem Labyrinthe der gekreuzten Seite in enger Verbindung stehen, lässt sich auf Grund meiner Versuche die coordinirte Innervation beinahe der ganzen Muskulatur analysiren.

Um mich eines recht anschaulichen Bildes zu bedienen, möchte ich den die Coordination der Muskulatur bewirkenden Apparat am liebsten mit einem Paar Zügel und dementsprechend die beiden Labyrinthe mit den zwei Händen des Kutschers vergleichen, welcher die Zügel hält und damit das ganze Gespann lenkt. Hält er beide Zügel gleichmässig gespannt, so ist der Fortgang des Gefährtes ein 
Ueb. den Zusammenhang zwischen der Muskulatur u. dem Labyrinth. 293

gerader. Lässt er jedoch z. B. links ein wenig nach und zieht dafür rechts stärker an, so wird sich das Gespann rechts wenden.

Mit der, diesem Bilde entsprechenden Reflexverbindung der Augenmuskeln mit dem Labyrinthe werde ich mich hier nicht weiter befassen, nachdem Professor Högyes diesen Gegenstand bereits erschöpft hat.

Sind die Halsmuskeln, welche den Kopf des Kaninchens um die Längs- und um die verticale Achse drehen, reehts in ebenso kräftiger Zusammenziehung als links, so befindet sich der Kopf in der Mittelstellung. Entferne ich nun das linke Labyrinth, oder schalte ich durch eine gewisse, durch Drehung hervorzurufende Körperhaltung die Wirking desselben beim gesunden Kaninchen aus, so sehe ich, dass nur die den Kopf nach links drehenden Muskeln in Thätigkeit sind, wogegen die rechtsdrehenden erschlaffen. Der Kopf ist in Folge dessen nach links gedreht. Das linke Labyrinth ist demnach mit den nach rechts drehenden Halsmuskeln, das rechte mit den nach links drehenden in Verbindung; diese Verbindung ist also eine gekreuzte.

Was nun die vorderen Extremitäten des Kaninchens betrifft, so besteht eine Verbindung derselben mit dem linken Labyrinth in Bezug auf die Adductoren der rechten und auf die Abductoren der linken vorderen Extremität und umgekehrt. Sind beide Labyrinthe unversehrt, und wirken beide in gleicher Weise, so werden die beiden vorderen Gliedmaassen in der Mitte, parallel der Längsachse des Körpers gehalten. Wirkt nun das linke Labyrinth nicht, sei es, weil es entfernt wurde, oder weil durch eine gewisse, durch Drehung des normalen Thieres erreichbare Körperhaltung die Wirkung desselben ausgeschaltet wurde, so verlassen die beiden vorderen Extremitäten die bisher innegehaltene "Mittelstellung", und beide werden nach rechts gehalten. (Adduction der linken, Abduction der rechten vorderen Extremität.)

Ich konnte des Ferneren auch beobachten, dass bei Drehung des normalen Kaninchens in der Sagittalebene nach vorne sich die beiden vorderen Extremitäten in Extension befinden und die Krallen auseinander gespreizt sind; wird das normale Thier in derselben Ebene nach rückwärts gedreht, so sind die vorderen Gliedmaassen in Beugestellung, die Krallen an einander liegend. Wird das unversehrte Kaninchen auf der horizontalen Drehscheibe nach rechts gedreht, so wenden sich die Sohlen beider vorderen Extremitäten nach 
rechts (Supination des linken, Pronation des rechten Fusses); bei Linksdrehung in derselben Ebene findet das Umgekehrte statt.

Nach einseitiger Operation konnte ich die Flexion und Extension Pronation und Supination nicht so genau beobachten wie die Abduction und die Adduction; da aber nach beiderseitiger Exstirpation der Labyrinthe alle diese Reflexbewegungen ausbleiben, ist es als sicher anzunehmen, dass die beiden Labyrinthe des Kaninchens auch mit der Flexion und Extension, sowie Pronation und Supination in Zusammenhang stehen. In Folge dessen kann ich theils auf Grund meiner Versuche, theils auf speculativem Wege in analogiam des von Professor Högyes für die Augenbewegungen aufgestellten Schemas behaupten, dass die Abductoren, Extensoren und Pronatoren mit dem gleichseitigen Labyrinthe, die Adductoren, Flexoren und Supinatoren aber mit dem Labyrinthe der gekreuzten Seite in Verbindung stehen.

Solange der Tonus der langen Muskeln, welche an beiden Seiten der Wirbelsäule wirken, der gleiche ist, bleibt die Wirbelsäule auch gerade. Fällt aber z. B. die Wirkung des linken Labyrinthes zu Folge Exstirpation desselben oder durch angemessene Drehung des Thieres aus, so erschlafft auch die an der linken Seite der Wirbelsäule befindliche Längsmuskulatur, die der rechten erhält zufolge ihres Tonus das Uebergewicht, und die Wirbelsäule krümmt sich nach rechts. Die Verbindung hier ist also nicht gekreuzt.

Die Coordination der unwillkürlichen Bewegungen des Kaninchens veranschaulicht nebenstehende schematische Zeichnung (Fig. 1).

Solange bei der Taube die Muskeln an beiden Seiten des Halses in gleicher Weise in Thätigkeit sind, befindet sich der Kopf in der "Mittelstellung". Werden beide Labyrinthe entfernt, so werden die Kopfbewegungen ungeordnet, das Thier hat die Herrschaft über die Bewegung seines Kopfes verloren. Entferne ich nur das linke Labyrinth, oder lasse ich in einer, durch bestimmte Drehung hervorzurufenden Körperhaltung nur das rechte Labyrinth in Thätigkeit, so wird sich der Kopf der Taube verdrehen, da sich nur die Muskeln von der linken Seite des Halses zusammenziehen, die der rechten hingegen erschlaffen. Das linke Labyrinth ist also mit den rechtsseitigen Halsmuskeln in Verbindung.

Sind beide Labyrinthe der Taube unversehrt, so wird diese mit beiden Füssen gleich weit ausschreiten. Sind beide Labyrinthe zerstört resp. entfernt, so knickt bald der eine, bald der andere Fuss ein. 


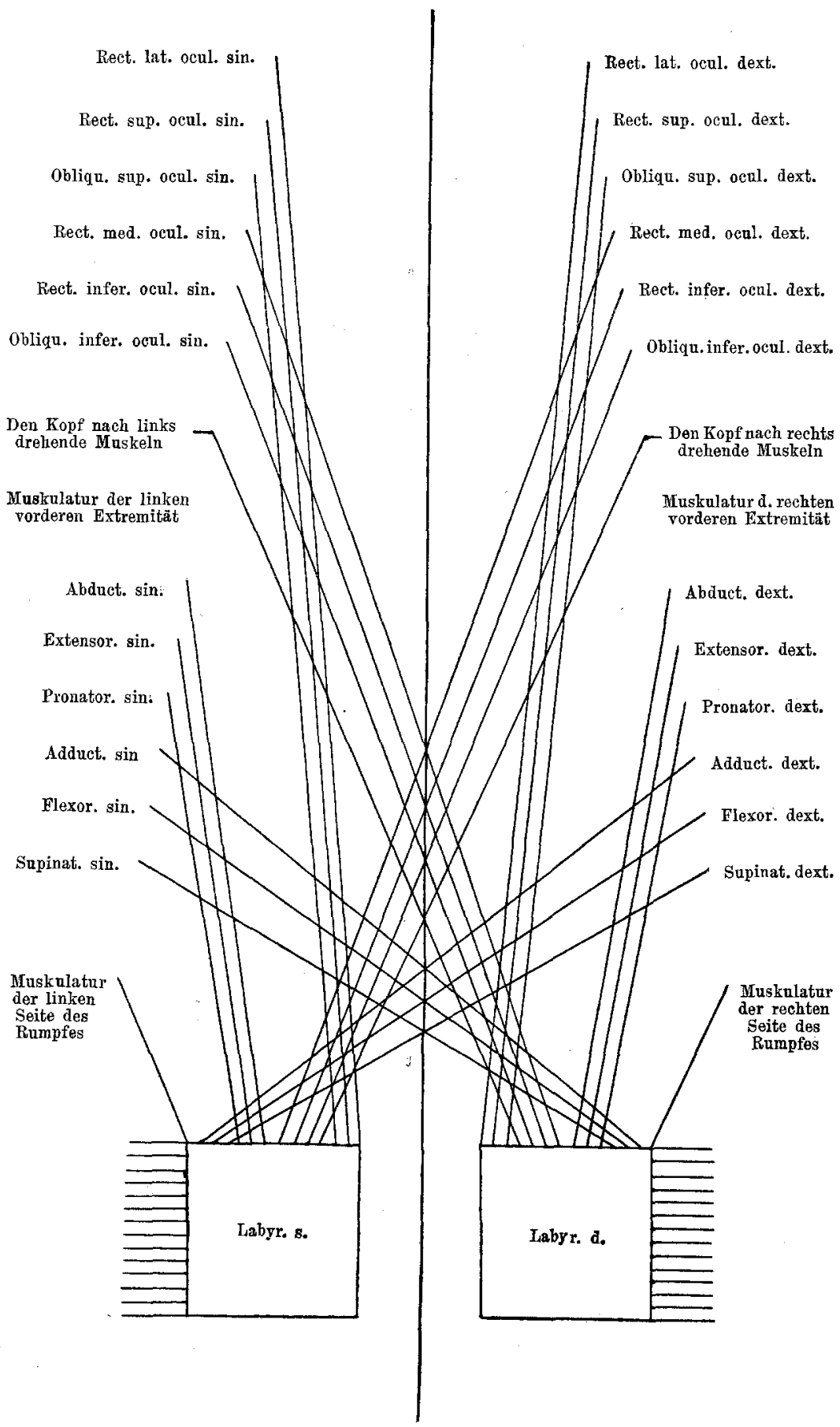

Fig. 1. Coordinationsschema der unwillkürlichen Bewegungen des Kaninchens. 
Wird bloss das linke Labyrinth entfernt, so schreitet die Taube mit dem rechten Bein $z u$ weit aus (beschreibt also einen Bogen nach links), während der linke Fuss fortwährend einknickt. Das.linke Labyrinth steht also mit der Reflexhemmungseinrichtung: des rechten und mit den Muskeln (Beuger, Strecker u. s. w.) des linken Beines in Verbindung.

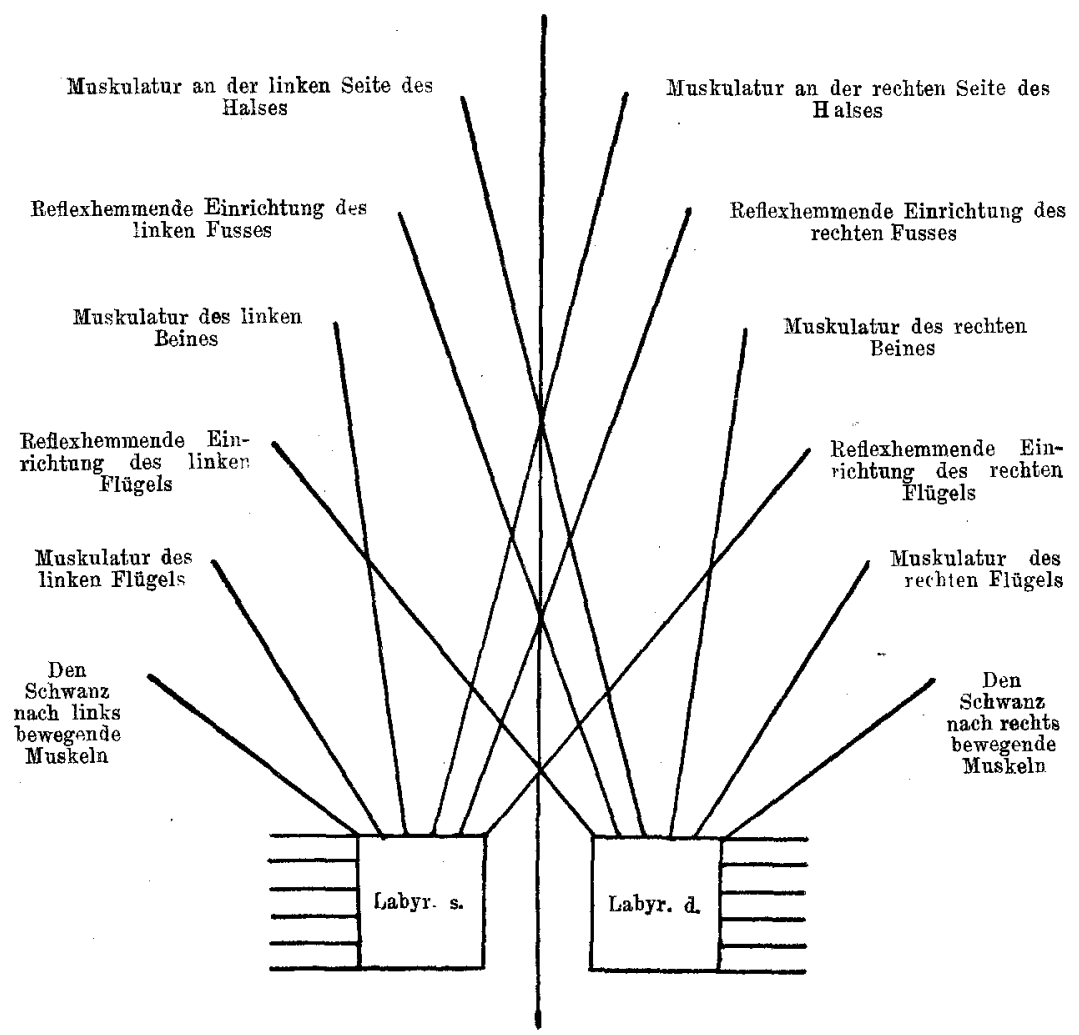

Fig. 2. Coordinationsschema der unwillkürlichen Bewegungen der Taube.

Werden beide Labyrinthe entfernt, so fliegt die Taube auch nach Jahren sehr unvollkommen. Ist bloss das linke Labyrinth entfernt, so ist der Flügelschlag links kraftlos, rechts dagegen übermässig. Die Verbindungen der Flügel mit den Labyrinthen sind also dieselben wie die der Beine.

Sind beide Labyrinthe der Taube unversehrt, so nimmt der Schwanz der Taube die "Mittelstellung" ein; wird nun das linke Labyrinth zerstört oder durch eine gewisse Drehung seine Wirkung ausgeschaltet, so dass nur das rechte Labyrinth in Wirksamkeit 
bleibt, so wird der Schwanz nach rechts gehalten. Jedes Labyrinth ist demnach mit den gleichseitigen Schwanzmuskeln in Verbindung. Die Coordination der unwillkürlichen Bewegungen der Taube veranschaulicht vorstehende schematische Zeichnung (Fig. 2).

Die Bewegangen der hinteren Extremitäten der Kaninchen vermochte ich vor der Hand nicht zu analysiren, weil ich die Thiere nicht in der Art zu fixiren vermochte, dass ihre hinteren Gliedmaassen hierbei frei geblieben wären. Die an hystero-epileptischen Kranken gemachten, oben erwähnten Beobachtungen Högy es' ergaben, dass mit der rechten oberen (vorderen) Extremität die linke untere associirte Bewegungen zeigte. Jedoch hat auch jene Anschauung ihre Berechtigung, dass sich die hinteren Gliedmaassen mit den gleichseitigen vorderen in gleichem Sinne bewegen. So zeigen z. B. bei der Taube Flügel und Bein der gleichen Seite associirte Bewegungen. Das halbseitig operirte Kaninchen wälzt sich darum nach der beschädigten Seite hin, weil (abgesehen von der Muskulatur des Rumpfes) beide Extremitäten der operirten Seite kraftlos einknicken und beide Gliedmaassen der unversehrten Seite in einer zu starken Thätigkeit sind. (Wir müssen eigentlich auch hier, ebenso wie bei den Tauben, die Existenz einer Reflexhemmungseinrichtung annehmen, welche z. B. nach Entfernung des linken Labyrinthes ibren Einfluss auf die rechtsseitige Muskulatur einstellt.) Auch bei den diesbezüglichen, mit Hülfe des inducirten Stromes vorgenommenen Untersuchungen verhielten sich die Extremitäten der gleichen Seite in gleicher Weise. Erwäge ich alle diese Beobachtungen, so muss ich vor der Hand die Coordination der Bewegungen der hinteren Gliedmaassen als offene Frage erklären.

Nach den Versuchen Högyes', später Dreyfuss', unterliegt es keinem Zweifel, dass auch die Athmungs- und die Phonationsmuskeln mit dem Labyrinthe in Verbindung stehen. Ob aber diese Verbindung eine gekreuzte ist oder nicht, das kann ich vor der Hand auch nicht entscheiden.

Die beiden Labyrinthe erhalten die to $\mathrm{n}$ is che Reflexinnervation der Muskulatur beider Körperhälften aufrecht. Erleidet eines der beiden oder erleiden beide Labyrinthe eine Beschädigung, so treten bei sämmtlichen Bewegungen des Körpers Störungen auf.

Schiff durchtrennte bei Hunden und Katzen auf der einen Seite die motorischen, auf der anderen die sensorisehen Nervenwurzeln des Rückenmarks, und fand, dass die Thiere mit den 
Extremitäten der einen Seite allzu grosse, ungeschickte Schritte machten, während die Gliedmaassen der anderen Seite gelähmt nachgezogen werden. Wenn ich aus der Aehnlichkeit dieser Resultate zu denen meiner Versuche einen Schluss ziehen darf, so möchte ich behaupten, dass jene Reflexhemmungseinrichtung, deren ich bei der Besprechung meiner Versuche an halbseitig operirten Kaninchen und Tauben bereits Erwähnung gethan, mit den sensorischen Nervenbahnen zusammenfällt. (Es wird dies auch durch meine mit Hülfe inducirter elektrischer Stromschläge ausgeführten Versuche wahrscheinlich gemacht.) Schreitet also z. B. eine linksseitig operirte Taube mit dem (ungeschickten) rechten Bein zu weit aus, so ist die sensorische Bahn dieses Fusses beschädigt; knickt das linke Bein kraftlos zusammen, so hat der motorische Nervenapparat dieser Extremität durch die Operation Schaden genommen. 